\title{
ON THE OSCILLATION OF THE DERIVATIVES OF A PERIODIC FUNCTION
}

\author{
BY \\ GEORGE POLYA AND NORBERT WIENER
}

1. Let $f(x)$ be a real valued periodic function of period $2 \pi$ defined for all real values of $x$ and possessing derivatives of all orders. Let $N_{k}$ denote the number of changes of sign of $f^{(k)}(x)$ in a period. We consider the order of magnitude of $N_{k}$ as $k \rightarrow \infty$.

(I) If $N_{k}=O(1), f(x)$ is a trigonometric polynomial.

(II) If $N_{k}=O\left(k^{\delta}\right)$ where $\delta$ is fixed, $0<\delta<1 / 2, f(x)$ is an entire function of finite order not exceeding $(1-\delta) /(1-2 \delta)$.

(III) If $N_{k}=o\left(k^{1 / 2}\right), f(x)$ is an entire function.

We prove this theorem by consideration of the Fourier series of $f(x)$

$$
f(x)=\sum c_{n} e^{i n x},
$$

$c_{-n}=\bar{c}_{n}(n=0,1,2, \cdots)$. Here, as in what follows, the sign $\sum$ without explicitly stated limits means a summation from $-\infty$ to $\infty$. Under the present conditions, the series (1) is absolutely and uniformly convergent for real $x$, and so are the Fourier series of $f^{\prime}(x), f^{\prime \prime}(x), \cdots$, obtained from (1) by term by term differentiation. If we focus our attention on the Fourier series, we may express the general trend of our theorem by saying that a small amount of oscillation in the higher derivatives implies a rapid decrease in the coefficients, this decrease being so extreme in case (I) that all coefficients from a certain point onward vanish.

The theorem we have to prove and a few analogous facts $\left({ }^{1}\right)$ point towards a general principle which cannot yet be stated in precise terms but which is not entirely unsuitably expressed by saying that a small amount of oscillation in the higher derivatives indicates a great amount of simplicity in the analytic nature of the function.

An analogous theorem may be formulated for almost periodic functions. As in other theorems of this kind, the number of changes of sign in a period is replaced by the density of these changes over the infinite line and a trigonometric polynomial is replaced by an entire function of exponential type. The extension of case (I) of our theorem offers the least difficulty.

Presented to the Society, May 2, 1941; received by the editors August 8, 1941.

(1) See S. Bernstein, Leçons sur les Propriétés Extrémales, Paris, 1926, pp. 190-197 and Communications de la Société Mathématique de Kharkow, (4), vol. 2 (1928), pp. 1-11; R. P. Boas and G. Pólya, Proceedings of the National Academy of Sciences, vol. 27 (1941), pp. 323325. 
2. We start with a few preliminary remarks on changes of sign. We consider first a real-valued function $f(x)$ which is defined in an interval $a \leqq x \leqq b$. We say that this function has $N$ changes of sign in this interval if it is possible to find $N+1$, and no more, abscissae $x_{0}, x_{1}, \cdots, x_{N}$ in the interval such that

$$
\begin{aligned}
x_{0}< & x_{1}<\cdots<x_{N-1}<x_{N}, \\
f\left(x_{\nu-1}\right) f\left(x_{\nu}\right) & <0, \quad \nu=1,2,3, \cdots, N .
\end{aligned}
$$

If a function has $N$ changes of sign in an interval, its derivative has there at least $N-1$ changes of sign. This variant of Rolle's theorem is easily proved by considering $\xi_{\nu}$, such that

$$
f\left(x_{\nu}\right)-f\left(x_{\nu-1}\right)=\left(x_{\nu}-x_{\nu-1}\right) f^{\prime}\left(\xi_{\nu}\right), \quad x_{\nu-1}<\xi_{\nu}<x_{\nu},
$$

and observing that $f\left(x_{\nu}\right) f^{\prime}\left(\xi_{\nu}\right)>0$ and that therefore

$$
f^{\prime}\left(\xi_{\nu-1}\right) f^{\prime}\left(\xi_{\nu}\right)<0, \quad \nu=2,3, \cdots, N .
$$

Applying this to the function $e^{a x} f(x)$ (where $a$ is a real constant) and its derivative $\left[e^{a x} f(x)\right]^{\prime}=e^{a x}\left[a f(x)+f^{\prime}(x)\right]$, we see that the number of changes of sign of

$$
(a+D) f(x)
$$

(where $D$ is the symbol of differentiation) is not inferior to $N-1, N$ being the number of changes of sign of $f(x)$.

Now let $f(x)$ be periodic with the period $2 \pi$. We say that the number of changes of sign of $f(x)$ in a period is $N$, if it is possible to find just $N+1$, and no more, abscissae $x_{0}, x_{1}, \cdots, x_{N}$ such that

$$
x_{N}=x_{0}+2 \pi \text {, }
$$

and (2), (3) hold. Observe that $f\left(x_{N}\right)=f\left(x_{0}\right)$ and that, therefore, $N$ is necessarily even. Hence it follows that the number of changes of sign of $(a+D) f(x)$ in a period is not inferior to that of $f(x)$. We defined $N_{k}$ in our initial statement; now we see that

$$
N_{0} \leqq N_{1} \leqq N_{2} \leqq \cdots \leqq N_{k-1} \leqq N_{k} \leqq \cdots .
$$

Observing that

we obtain :

$$
(a+D)(a-D) \sum \frac{c_{n} e^{i n x}}{a^{2}+n^{2}}=\sum c_{n} e^{i n x}
$$

Lemma I. The number of changes of sign of the function (1) in a period is not inferior to that of

$$
\sum \frac{c_{n} e^{i n x}}{a^{2}+n^{2}}
$$


3. The series (1) represents a trigonometric polynomial of order $m$ if $c_{n}=0$ for $n=m+1, m+2, \cdots$. If $f(x)$ is a trigonometric polynomial of order $m$, it cannot have more than $2 m$ roots in a period; this is well known. Observe that for large $k, f^{(k)}(x)$ has actually $2 m$ changes of sign, because as $k \rightarrow \infty$, $(i m)^{-k} f^{(k)}(x)$ approaches the first or the second of the two expressions

$$
-c_{-m} e^{-i m x}+c_{m} e^{i m x}, \quad c_{-m} e^{-i m x}+c_{m} e^{i m x},
$$

according as $k$ is odd or even. The second of these expressions is of the form $2\left|c_{m}\right| \cos (m x-\gamma)$, with a certain real $\gamma$ and the first is of the same form except for a factor $i$.

The case (I) of our theorem characterizes the trigonometric polynomials and can be stated as follows: $A$ real-valued periodic function $f(x)$ possessing derivatives of all orders is a trigonometric polydomial if and only if the number of changes of sign of $f^{(k)}(x)$ remains bounded for $k \rightarrow \infty$.

In order to prove this we consider (1). We have to show that some $f^{(k)}(x)$ have an arbitrarily great number of changes of sign if there are $c_{n} \neq 0$ with arbitrarily large subscripts $n$. More precisely we shall show this:

If $m>0$ and $c_{m} \neq 0$, then all derivatives of (1), from a certain stage onward, have not less than $2 m$ changes of sign.

In fact, by repeated application of Lemma I, we ascertain that

$$
f^{(k)}(x)=i^{k} \sum n^{k} c_{n} e^{i n x}
$$

does not have fewer changes of sign than

$$
i^{k} \sum\left(\frac{2 m n}{m^{2}+n^{2}}\right)^{k} c_{n} e^{i n x}
$$

But since it is given that $c_{m} \neq 0$ and that $\sum c_{n}$ is absolutely convergent, we have from a certain $k$ onward

$$
\left|c_{m}\right|>\left(\sum_{n=1}^{m-1}+\sum_{n=m+1}^{\infty}\right)\left(\frac{2 m n}{m^{2}+n^{2}}\right)^{k}\left|c_{n}\right| .
$$

Indeed we have for $n>0, n \neq m, 0<2 m n<m^{2}+n^{2}$, and therefore, each term tends to 0 on the right-hand side of $(8)$ for $k \rightarrow \infty$.

But if (8) holds for a certain even $k$, the sum in (7) has the same sign as the second expression (5) in all those real points $x$ in which this latter reaches $2\left|c_{m}\right|$, the maximum of its absolute value. This maximum is reached with alternating signs, in equidistant points, the distance of two consecutive points being $\pi / m$. Therefore (7) has not less than $2 m$ changes of sign. We have proved this for even $k$ but the same is true and the proof is nearly the same for odd $k$. Then, by Lemma I, (6) has not less than $2 m$ changes of sign, and case (I) of our theorem is proved.

4. We consider the case (III) of our theorem before case (II). 
If the periodic function $f(x)$ is analytic along the whole real axis, it is analytic in a certain horizontal strip bisected by the real axis and the Fourier series (1), which is a Laurent series in

$$
z=e^{i x}
$$

converges in the interior of the strip. Hence, by examining

$$
\limsup _{n \rightarrow \infty}\left|c_{n}\right|^{1 / n}
$$

we can distinguish the following three cases:

If $f(x)$ is not analytic along the whole real axis, (9) has the value 1.

If $f(x)$ is analytic in a certain horizontal strip of width $2 h$ bisected by the real axis, but in no wider horizontal strip, (9) has the value $e^{-h}$.

If $f(x)$ is an entire function, (9) has the value 0 .

In order to prove case (III) of our theorem, we have to show that in the first two cases $N_{k}=o\left(k^{1 / 2}\right)$ is excluded. We prove the following statement.

If there exists a positive number $\gamma$ such that

$$
\limsup _{n \rightarrow \infty}\left|c_{n}\right| e^{n \gamma}=\infty \text {, }
$$

then there exists a positive number $g$ such that $f^{(k)}(x)$ has, for an infinity of values of $k$, not less than $(k / g)^{1 / 2}$ changes of sign.

By the considerations of the foregoing section, $f^{(k)}(x)$ has certainly not less than $2 m$ changes of sign if (8) holds. Using (10), we have to find an arbitrarily large $m$ and a corresponding $k$ such that (8) holds. We shall succeed in finding such an $m$ by applying the following known lemma $\left({ }^{2}\right)$.

LEMMA II. We consider two infinite sequences $l_{1}, l_{2}, \cdots, l_{n}, \cdots$ and $s_{1}, s_{2}, \cdots, s_{n}, \cdots$, and suppose that

$$
\begin{gathered}
l_{n} \geqq 0, \\
0<s_{1}<s_{2}<s_{3}<\cdots, \\
\lim _{n \rightarrow \infty} l_{n}=0, \\
\limsup _{n \rightarrow \infty} l_{n} s_{n}=\infty .
\end{gathered}
$$

Then there exists an infinity of integers $m$ such that

$$
\begin{array}{cr}
l_{m} \geqq l_{m+\mu}, & \mu=1,2,3, \cdots, \\
l_{m} s_{m} \geqq l_{m-\mu} s_{m-\mu}, & \mu=1,2, \cdots, m-1 .
\end{array}
$$

We put

(2) See G. Pólya and G. Szegö, Aufgaben und Lehrsätze aus der Analysis, Berlin, 1925, vol. 1, pp. 18 and 173, Problem 109. 


$$
\left|c_{n}\right|=l_{n}, \quad e^{n \gamma}=s_{n} .
$$

This choice satisfies (11), (12), (13), (14); in fact, (13) is satisfied because (1) is convergent, and (14) is satisfied because we have supposed (10). Thus we obtain an infinity of $m$ such that

$$
\begin{aligned}
\left|c_{m+\mu}\right| & \leqq\left|c_{m}\right|, & \mu=1,2,3, \cdots, \\
\left|c_{m-\mu}\right| e^{(m-\mu) \gamma} & \leqq\left|c_{m}\right| e^{m \gamma}, & \mu=1,2, \cdots, m-1 .
\end{aligned}
$$

This we use to estimate the following sum. (Our ultimate aim is to prove (8).)

$$
\begin{aligned}
& \sum_{\mu=1}^{m-1}\left(\frac{2 m(m-\mu)}{m^{2}+(m-\mu)^{2}}\right)^{k}\left|\frac{c_{m-\mu}}{c_{m}}\right|+\sum_{\mu=1}^{\infty}\left(\frac{2 m(m+\mu)}{m^{2}+(m+\mu)^{2}}\right)^{k}\left|\frac{c_{m+\mu}}{c_{m}}\right| \\
& <\sum_{\mu=1}^{m-1}\left(\frac{2 m(m-\mu)}{m^{2}+(m-\mu)^{2}}\right)^{k} e^{\gamma \mu}+\sum_{\mu=1}^{\infty}\left(\frac{2 m(m+\mu)}{m^{2}+(m+\mu)^{2}}\right)^{k} \\
& =S_{1}+S_{2} .
\end{aligned}
$$

We introduced the abbreviations

$$
\begin{aligned}
& S_{1}=\sum_{\mu=1}^{m-1} e^{\gamma \mu}\left(1+\frac{\mu^{2}}{2 m(m-\mu)}\right)^{-k}, \\
& S_{2}=m \sum_{\mu=1}^{\infty}\left(\frac{2(1+\mu / m)}{1+(1+\mu / m)^{2}}\right)^{k} \frac{1}{m},
\end{aligned}
$$

and we shall consider $S_{1}$ and $S_{2}$ in turn.

(1) Split the sum $S_{1}$ in two parts, $\mu$ being less than or equal to $m / 2$ in the first part and greater than $m / 2$ in the second. Using the fact that

$$
(1+x)^{-1}<e^{-x / 2}, \quad 0<x<1 \text {, }
$$

we obtain

We put

$$
\begin{aligned}
S_{1} & <\sum_{1}^{m / 2} e^{\gamma \mu-k \mu 2 / 4 m^{2}}+\sum_{m / 2}^{m-1} e^{\gamma \mu}(4 / 5)^{k} \\
& <\sum_{1}^{m / 2} e^{-[(k / 4 m 2)-\gamma] \mu}+m e^{\gamma m}(4 / 5)^{k} \\
& <e^{-(o-\gamma)} /\left(1-e^{-(o-\gamma)}\right)+m e^{\gamma m}(4 / 5)^{4 o m 2} .
\end{aligned}
$$

$$
k=4 g m^{2}
$$

where $g$ is a positive integer, $g>\gamma$. We choose a fixed $g$ such that for suffciently great $m$

$$
S_{1}<1 / 2
$$


(2) The function $2 x\left(1+x^{2}\right)^{-1}$ decreases for $x>1$. Therefore by (17)

$$
S_{2}<m \int_{1}^{\infty}\left(\frac{2 x}{1+x^{2}}\right)^{k} d x \sim \frac{m}{2}\left(\frac{2 \pi}{k}\right)^{1 / 2}=\frac{1}{2}\left(\frac{\pi}{2 g}\right)^{1 / 2} .
$$

We used a well known asymptotic evaluation of definite integrals $\left({ }^{3}\right)$ and (18). If $g \geqq 2$, which we assume, we obtain for sufficiently great $m$

$$
S_{2}<1 / 2 \text {. }
$$

But (15), (19), (20) show that (8) is true so that $f^{(k)}(x)$ has not fewer changes of sign than

$$
2 m=(k / g)^{1 / 2} .
$$

5. We now proceed to the proof of case (II).

LEMMA III. The Fourier series (1) represents an entire function of the finite order $\lambda, \lambda>1$, if and only if

$$
\liminf _{n \rightarrow \infty} \frac{\log \log \left(1 /\left|c_{n}\right|\right)}{\log n}=\frac{\lambda}{\lambda-1} .
$$

The proof consists of two parts. Both parts follow familiar lines; so we do not give all the details.

(1) Assume that $f(x)$ is entire and of order $\lambda$. Then for a fixed positive $\epsilon$ and for sufficiently great $|x|$,

$$
|f(x)|<e^{|x|^{\lambda+e}}
$$

If we evaluate $c_{n}$ and shift the line of integration (periodicity and Cauchy's formula), we obtain as a result that if $r$ is any positive number,

$$
\begin{aligned}
c_{n} & =\frac{1}{2 \pi} \int_{-i r-\pi}^{-i r+\pi} f(x) e^{-i n x} d x \\
& =\frac{1}{2 \pi} e^{-n r} \int_{-\pi}^{\pi} f(-i r+u) e^{-i n u} d u, \\
\left|c_{n}\right| & \leqq e^{(r+\pi)^{\lambda+\epsilon_{-n r}}}
\end{aligned}
$$

Here we use (22). We choose $r$, for given $n$, so that this right-hand side of (23) shall be a minimum. It follows by straight-forward calculation that (21) holds with " $\geqq$ " instead of " $=$."

(2) Assume that

$$
\liminf _{n \rightarrow \infty} \frac{\log \log \left(1 /\left|c_{n}\right|\right)}{\log n}=\kappa>0 .
$$

(3) See, for example, G. Pólya and G. Szegö, loc. cit., vol. 1, pp. 78 and 244, Problem 201. 
Therefore we have, for a given positive $\epsilon$ and all sufficiently great $n$

$$
\left|c_{n} e^{i n x}\right|<e^{-n^{\kappa-\epsilon}+|x| n} \text {. }
$$

We choose $n$, for a given $x$, so that the right-hand side is a maximum. This maximum gives the right order of magnitude because the terms of (1) whose index surpasses a certain multiple of the index of the maximum term, yield a negligible contribution. We find that the order $\lambda$ of $f(x)$ satisfies the inequality

$$
\lambda \leqq \frac{\kappa}{\kappa-1}
$$

This gives (21) with "ฏ” instead of " =."

6. Now we are prepared to prove case (II) of our theorem. We have to show that if the entire function $f(x)$ is of order $\lambda$, and $\epsilon>0$ then, for an infinity of $k$,

$$
N_{k}>k^{(\lambda-1) /(2 \lambda-1)-\epsilon} \text {. }
$$

Put $\lambda /(\lambda-1)+\eta=\gamma, \eta$ being positive and small. By Lemma III, the fact we have to show can be stated as follows:

If there exists a positive number $\gamma, \gamma>1$, such that

$$
\limsup _{n \rightarrow \infty}\left|c_{n}\right| e^{n \gamma}=\infty
$$

then there exists a positive $g$ such that $f^{(k)}(x)$ has, for an infinity of $k$, more than $(k / g)^{1 /(\gamma+1)}$ changes of sign.

We apply Lemma II, whose conditions are satisfied by

$$
l_{n}=\left|c_{n}\right|, \quad s_{n}=e^{n \gamma} .
$$

We obtain the result that for an infinity of $m$,

$$
\begin{array}{rlr}
\left|c_{m}\right| & \geqq\left|c_{m+\mu}\right|, & \mu=1.2, \cdots, \\
\left|c_{m}\right| e^{m^{\gamma}} & \geqq\left|c_{m-\mu}\right| e^{(m-\mu) \gamma}, & \mu=1,2, \cdots, m-1 .
\end{array}
$$

Hence, using the fact that $\gamma>1$, we obtain

$$
\begin{aligned}
& \sum_{\mu=1}^{m-1}\left(\frac{2 m(m-\mu)}{m^{2}+(m-\mu)^{2}}\right)^{k}\left|\frac{c_{m-\mu}}{c_{m}}\right|+\sum_{\mu=1}^{\infty}\left(\frac{2 m(m+\mu)}{m^{2}+(m+\mu)^{2}}\right)^{k}\left|\frac{c_{m+\mu}}{c_{m}}\right| \\
& \quad<\sum_{\mu=1}^{m-1}\left(\frac{2 m(m-\mu)}{m^{2}+(m-\mu)^{2}}\right)^{k} e^{\gamma m^{\gamma-1} \mu}+\sum_{\mu=1}^{\infty}\left(\frac{2 m(m+\mu)}{m^{2}+(m+\mu)^{2}}\right)^{k} \\
& =S_{1}^{\prime}+S_{2} .
\end{aligned}
$$

$S_{2}$ has the same meaning as before (see (17)), and 
(25)

$$
\begin{aligned}
S_{1}^{\prime} & =\sum_{\mu=1}^{m-1} e^{\gamma m^{\gamma-1} \mu}\left(1+\frac{\mu^{2}}{2 m(m-\mu)}\right)^{-k} \\
& <\sum_{\mu=1}^{m / 2} e^{\gamma m^{\gamma-1} \mu-k \mu 2 / 4 m^{2}}+m e^{\gamma m^{\gamma}}(4 / 5)^{k} \\
& =\sum_{\mu=1}^{m / 2} e^{-m^{\gamma-1}(g \mu 2-\gamma \mu)}+m e^{\gamma m \gamma}(4 / 5)^{4 o m} \gamma+1
\end{aligned}
$$

We put

$$
k=4 g m^{\gamma+1},
$$

and we choose $g$ so that

$$
\gamma+1 \leqq g<\gamma+2,
$$

and so that $k$ is an integer. This choice assures that

$$
S_{1}^{\prime} \rightarrow 0, \quad S_{2} \rightarrow 0
$$

for $m \rightarrow \infty$ (see (25) and the considerations preceding (20)). Therefore, by (24), $(8)$ is true and $f^{(k)}(x)$ has not fewer changes of sign than

$$
2 m>2[k / 4(\gamma+2)]^{1 /(\gamma+1)} \text {. }
$$

Brown UnIVersity,

Providence, R. I.

Massachusetts Institute of Technology,

Cambridge, Mass. 